\title{
Subtemporal Extradural Approach for Dehiscence of the Superior Semicircular Canal: Surgical Technique and Results in Three Consecutive Patients
}

\author{
Sarah Hendrickx¹, Abdulhamid Ciçek ${ }^{1}$, Jeroen Cortier ${ }^{1}$, Olivier Van Damme ${ }^{1}$, \\ Jeroen Van Lerbeirghe ${ }^{1}$, Pieterjan Leupe ${ }^{2,3}$, Glen Forton ${ }^{2,3}$, Dimitri Vanhauwaert ${ }^{1,3}$ \\ ${ }^{1}$ Department of Neurosurgery, Roeselare, Belgium \\ ${ }^{2}$ Department of Otorhinolaryngology, Head-Neck-Surgery, Roeselare, Belgium \\ ${ }^{3}$ Multidisciplinary Skull Base Centre, Roeselare, Belgium \\ Email: sarahhendrickx@outlook.com,dimitri.vanhauwaert@azdelta.be
}

How to cite this paper: Hendrickx, S., Ciçek, A., Cortier, J., Van Damme, O., Van Lerbeirghe, J., Leupe, P., Forton, G. and Vanhauwaert, D. (2022) Subtemporal Extradural Approach for Dehiscence of the Superior Semicircular Canal: Surgical Technique and Results in Three Consecutive Patients. Open Journal of Modern Neurosurgery, 12, 28-38.

https://doi.org/10.4236/ojmn.2022.121003

Received: June 17, 2021

Accepted: January 4, 2022

Published: January 7, 2022

Copyright $\odot 2022$ by author(s) and Scientific Research Publishing Inc. This work is licensed under the Creative Commons Attribution International License (CC BY 4.0).

http://creativecommons.org/licenses/by/4.0/ (c) (i) Open Access

\begin{abstract}
We describe three patients with severe disabling symptoms of unilateral dehiscence of the superior semicircular canal (DSSC) who had surgical treatment. Each patient underwent a unilateral subtemporal extradural approach with resurfacing the DCCS. In all 3 patients, all symptoms were completely resolved and remained symptom free on the long term. There were no postoperative complications. Only one patient experienced a temporary CSF hypotension syndrome and some dizziness. The pseudo-conductive hearing loss improved or resolved in all patients. Surgical treatment should be considered in patients with severe, disabling DSSC symptoms. Surgical resurfacing of the DSSC is a safe and rewarding surgical technique. The long term success rate regarding the elimination of the pseudo-conductive hearing loss and resolution of vestibular symptoms outweigh the potential surgical risks of this technique in these patients.
\end{abstract}

\section{Keywords}

Superior Semicircular Canal Dehiscence, Subtemporal Craniotomy, Minor Syndrome

\section{Introduction}

Dehiscence of the superior semicircular canal (DSSC) causing otovestibular symptoms is quite a rare diagnosis. This phenomenon was first described by the 
American physician Lloyd B. Minor in 1998 in the Journal of Otology and Neurotology [1]. Although precise epidemiological data are not available, cadaver studies showed a prevalence of SSCD in $0.7 \%$ of the 1000 temporal bone examined from 596 specimens [2]. The incidence of SSCD increases with age: most patients are middle-aged at the time of diagnosis [1].

Five core symptoms have been described: Tullio's phenomenon, Hennebert's sign, pseudo-conductive hearing loss, pulsatile tinnitus and autophony.

Tullio's phenomenon consists of sound-induced vertigo, nausea and nystagmus. Hennebert's sign, on the other hand, is the occurrence of nystagmus, vertigo and nausea secondary to an increase of intracranial pressure (caused by coughing, sneezing or Valsalva). All of these symptoms are not necessarily present in every patient with SSCD [1] [2] [3] [4] [5]. The nystagmus typically beats in the plane of the involved dehiscent canal (according to Ewald's first law). In major defects, with associated hypofunction of the attained vestibulum, the nystagmus may also beat in the direction of the other semicircular canals, but this is rare. In addition, there are also several rather non-specific vestibular symptoms that may occur, such as visual vertigo and oscillopsia, pulsatile tinnitus, gait instability, headache, sensorineural hearing loss and aural fullness. The clinical picture is therefore very variable [1] [2] [3] [4] [5]. Nevertheless, most patients present with vestibular complaints.

Clinically, the differential diagnosis must be made with otosclerosis, Menière's disease, perilymph fistula, acute vestibular neuritis and other dehiscence syndromes.

Careful clinical examination and a high-resolution CT scan with double oblique reconstructions are mandatory to rule out a DSSC before even considering a stapedotomy in case of suspected otosclerosis [1] [2] [3] [4] [5]. In a series of 150 ears with clinical suspicion of otosclerosis, DSSC was detected by CT imaging in $5.3 \%$ of cases [4].

The precise etiology of DSSC is still unknown, although several mechanisms have been put forward. Presumably, a combination of these mechanisms results in the occurrence of DSSC. Firstly, congenital predisposition has been proposed, suggesting a thinner tegmen tympani at birth (first hit). Secondly, insufficient ossification and thickening of the tegmen tympani during the first three years of life (second hit) and, finally, erosive processes can further weaken the tegmen tympani (third hit). The defect may also be due to extreme thinning of the bone, eventually allowing motion of the involved segment. This is described as the "near dehiscence syndrome". Examples of these erosive processes are infection, increased intracranial pressure, tumors, fractures of the temporal bone. Cases have been described in which cochlear and other semicircular canal deficiencies were caused by a high riding jugular bulb, the superior petrous sinus, the internal carotid artery [6], Pacchioni's granulations and, finally, even the brain itself. Even mild head trauma or a sudden increase in intracranial pressure can cause the final crack, exposing the perilymphatic space [1]-[6]. A genetic cause has also been 
described as part of Usher's syndrome (CDH23 pathogenic variant) [3].

In normal conditions, the inner ear is separated from the intracranial space by a bony barrier, i.e. every part of the membranous labyrinthi is covered by solid bone (the otic capsule), thus effectively blocking the transfer of pressure waves between the middle fossa and inner ear. An osseous defect would allow pressure waves to propagate in and out of the membranous labyrinth. This is the basis of the "third window" theory. Leakage of acoustic energy to the intracranial space, causes the pseudo-conductive hearing loss found at pure tone audiometry. Conversely, when measuring bone conduction, Tonndorff's compressional mechanism plays an important role: as the fluids in the cochlea are compressed, both round window membrane and stapes are pushed outward, thus creating a pressure difference in the cochlear partitions due to differences in impedance between scala tympani and scala vestibule.

In case of a dehiscence, the inequality in impedance between the scalae is increased, hence the pressure difference across the cochlear partition is increased, resulting in "improved" bone conduction thresholds, thus contributing to the pseudo-conductive hearing loss [1]-[7].

Performing a Valsalva maneuver has 2 effects. Firstly, the generation of a relative overpressure in the middle ear cavity leads to an inward displacement of the both stapes and round window (exciting). Simultaneously, increased intrathoracic pressure,reduced venous return and increased intracranial pressure occur. At the dehiscence the membrane pushed inwards; resulting in an ampullafugal movement of the endolymph (inhibitory). Therefore, both components of a Valsalva maneuver may cause Hennebert's Sign [5].

Typically, neurological examinations are normal. The audiogram typically shows a predominantly low-frequency pseudo-conductive hearing loss, composed of elevated air-conduction thresholds and better-than-normal bone conduction thresholds. Tympanogram is normal and acoustic reflexes are typically present, while in cases of true stapes fixation due to otosclerosis, acoustic reflexes are obligatory absent [3] [4] [5]. The Weber test lateralizes to the DSSC side, but this is also the cases with true otosclerosis [6].

The eye movements and nystagmus are examined using Frenzel's glasses, or better still, using infrared videonystagmoscopy. During examination, auditory stimuli are administered and Valsalva's maneuver is performed in order to objectivate the presence Tullio's sign and Hennebert's sign. Also, VEMP recording is performed (Vestibular Evoked Myogenic Potentials). The VEMP amplitude is typically very much increased in case of a DSSC [3] [4] [5]. VEMP recording also allows to differentiate with true otosclerosis, since the VEMP is obligatory absent due to inadequate mobility of the ossicular chain [4] [5]. High Definition Computerized tomography (CT) of the temporal bone with thin sections of 0.5 $\mathrm{mm}$ or less are essential in diagnosing DSSC. Specific reconstructions in the plane of and perpendicular to the SCC, so calles "double oblique" reconstructions are needed [3] [4] [5]. Only when the dehiscence is visible in both recon- 
struction planes can the radiological diagnosis be confirmed. A very thin layer of bone can be missed on CT and give a false picture of dehiscence [1] [2] [3] [4] [5]. The size of the dehiscence is usually significantly overestimated on CT [8]. It is the combination of both the clinical symptoms and the technical exams that yield the final diagnosis of DSSC [1] [2] [3] [4] [5] [8]. Magnetic resonance imaging (MRI) is advised to exclude concomitant pathology, such as a Chiari malformation, but does not contribute to the diagnosis of DSSC [3] [4].

Surgical intervention can significantly improve the quality of life in patients, with disabling symptoms. Three surgical techniques can be used in the treatment and are described in the Discussion section [3] [16] [17].

\section{Methods}

We describe the retrospective data of three patients with severe disabling DSSC symptoms, summarized in Table 1. All patients had a unilateral dehiscence, 2 on the right and 1 on the left side. There were no bilateral dehiscences. Patients were between the age of 38 and 54 years and 1/3 was female. No relevant medical history could be retained except for a bilateral paracenthesis during childhood in patient 3. No triggering factors could be noted. Patients had symptoms of Tullio's phenomenon (3/3), Hennebert's sign (3/3), autophony (3/3), pulsatile tinnitus (2/3), instability (3/3) and pseudo-conductive hearing loss (3/3) (Table 2). The severe reduction in quality of life caused a major disruption of their daily routine.

Otoscopic examination showed no abnormalities (Table 3). Audiometry

Table 1. Individual charachteristics of 3 patients with SCCD.

\begin{tabular}{|c|c|c|c|c|c|c|c|c|}
\hline Patient & Sex & Age at operation (years) & History & Unilateral or bilateral DCSS & Side & Procedure & ELD & Mannitol \\
\hline 1 & male & 43 & 0 & unilateral & Right & craniotomy & + & + \\
\hline 2 & female & 54 & 0 & unilateral & left & craniotomy & + & + \\
\hline 3 & male & 38 & paracenthesis & unilateral & right & craniotomy & + & + \\
\hline
\end{tabular}

Table 2. Patients' symptoms before surgical repair.

\begin{tabular}{ccccccc}
\hline Patient & Conductivehearing loss & Tullio's phenomenon & Hennebert's Sign & Autophony & Pulsatile tinnitus & Instability \\
\hline 1 & + & + & + & + & + & + \\
2 & + & + & + & + & + & + \\
3 & + & 3 & 3 & 3 & 0 & + \\
Total & 3 & & + & 2 & 3 \\
\hline
\end{tabular}

Table 3. Neuro-otological exams of the 3 patients.

\begin{tabular}{ccccccc}
\hline \multirow{2}{*}{ Patient } & \multirow{2}{*}{ Otoscopy } & Audiometry & $\begin{array}{c}\text { Air-Bone } \\
\text { Gap }\end{array}$ & Tympanometry & $\begin{array}{c}\text { Acoustic } \\
\text { Reflexes }\end{array}$ & VEMP \\
\hline 1 & normal & Pseudo-conductive hearing loss & $20 \mathrm{db}$ & type A & present increased amplitude dehiscence \\
2 & normal & Pseudo-conductive hearing loss & $30 \mathrm{db}$ & type A & present increased amplitude dehiscence \\
3 & normal & Pseudo-conductive hearing loss & $20 \mathrm{db}$ & type A & present increased amplitude dehiscence \\
\hline
\end{tabular}


showed low-frequency pseudo-conductive hearing loss of 20 - $30 \mathrm{~dB}$. Figure 1 shows the preoperative audiogram of patient 1 . Normal middle ear aeration (type A) could be demonstrated on tympanogram. Acoustic reflexes were present. VEMPs showed an increased amplitude. CT demonstrated a clear DSSC in each of the patients (Figure 2).

\section{LINKER OOR}

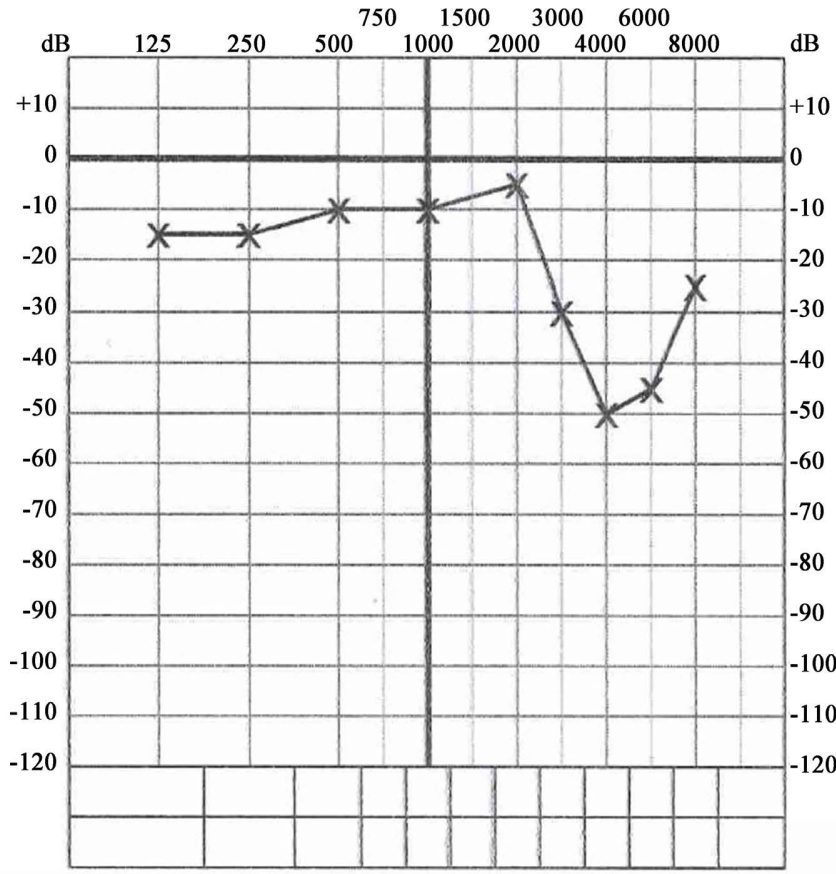

RECHTER OOR

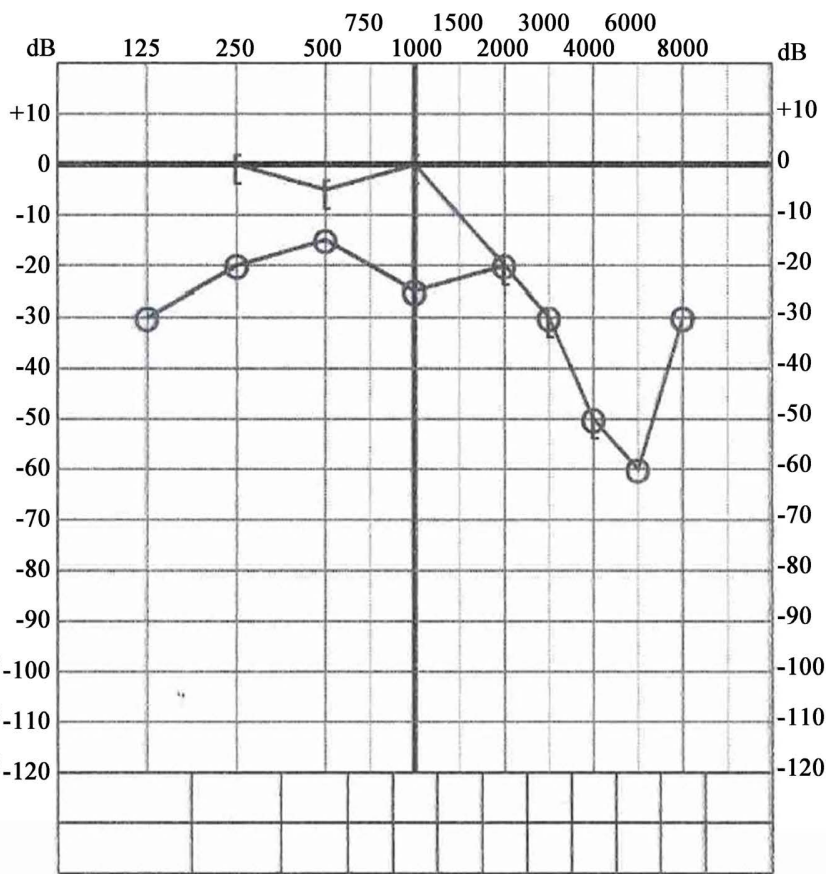

Figure 1. Preoperative Audiogram of Patient 1 shows typical low-frequency pseudo-conductive hearing loss with loss of air conduction $(\mathrm{O})$ and preserved bone conduction ([) on the affected side (right ear) comparing to the normal side (left ear).

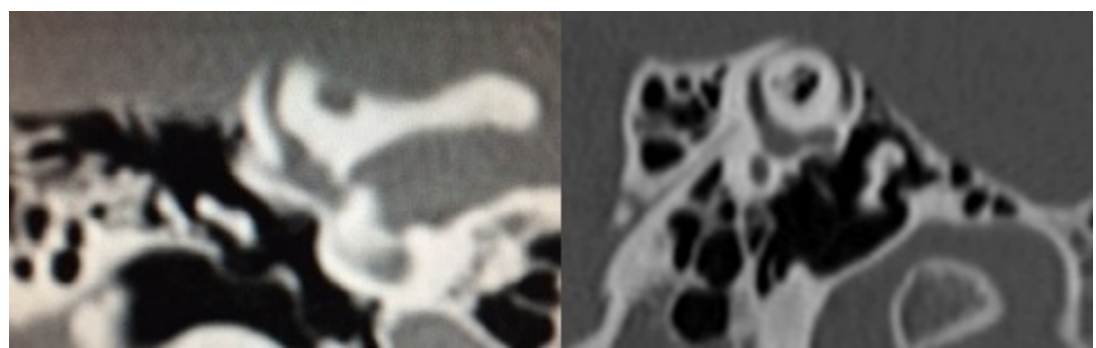

Figure 2. Preoperative Computed Tomography image of the right temporal bone of patient 1 in the plane and perpendicular to the Superior Semicircular Canal. The image shows the absence of bony coverage of the Superior Semicircular Canal.

All three patients underwent surgical treatment between 2011 and 2018. The procedures were performed by the same neurosurgeon (DV). 2 Otolaryngologists (PL and GF) were involved. An external lumbar drainage (ELD) was administered preoperatively and mannitol was administered to each patient to minimize brain retraction. Each patient underwent a subtemporal craniotomy. A C-shaped incision was made from pre- to postauricular. After removal of the bone flap, an extradural access was dissected up to the eminentia arcuata with 
the tegmen tympani. In each patient, a clear defect in the tympani was visible peroperatively. The intraoperative microscopic view of the Eminentia Arcuata with dehiscence (arrow) of the SSC of patient 3 is visible in Figure 3. The DSSC was covered with bone or cartilage and a tensor fascia lata plasty. The fragments were fixed with fibrin glue (Figure 4). Patients were observed in the hospital for several days with a normal recovery in each of the three patients.

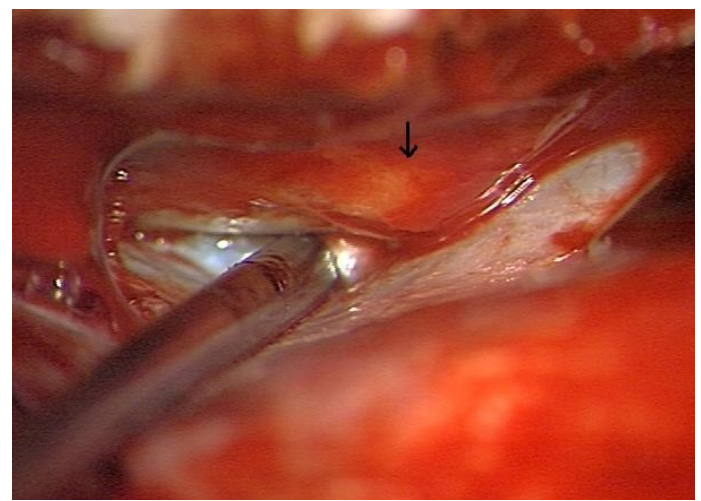

Figure 3. Intraoperative microscopic view of the Eminentia Arcuata with dehiscence (arrow) of the SSC of patient 3.

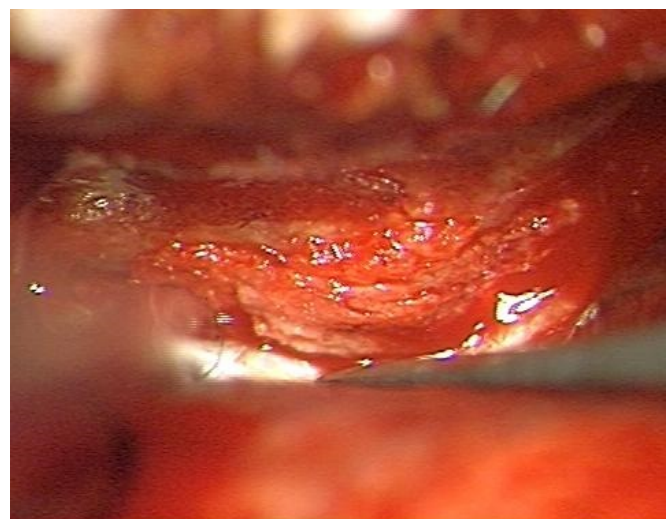

Figure 4. Intraoperative microscopic view of the Eminentia Arcuata showing coverage of the SCC by bone and tensor fascia latae plasty.

\section{Results}

In two out of three patients, all symptoms were resolved immediately. The third patient experienced a significant improvement and maintained a minimal dizziness after surgery. Long term follow-up showed full relief of all symptoms. There were no postoperative complications, except temporary complaints of CSF hypotension and dizziness in one patient. Subjectively, this patient had slightly impaired hearing after the procedure, which also resolved spontaneously. No new interventions were necessary. The hearing normalized in all patients as compared to their preoperative status (Figure 5). The subjective quality of life improved enormously for the patients. Long-term follow-up averaged 5.06 years (2.47 - 8.15 years). The permanent result was confirmed in all 3 patients. 

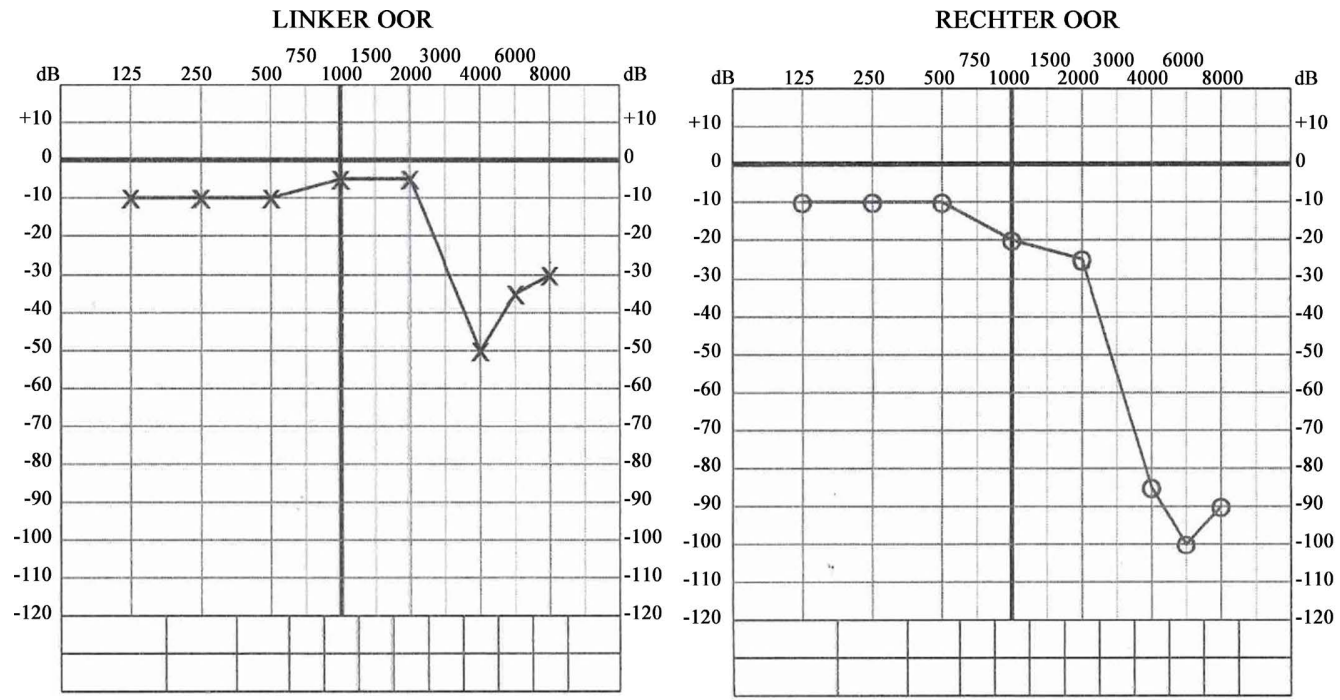

Figure 5. Postoperative Audiogram of Patient 1 shows a similar result between the affected side (right ear) and the normal side (left ear). The pseudo-conductive hearing loss with the typical air-bone gap has disappeared.

\section{Discussion}

Some patients present with either predominantly auditory or vestibular symptoms, or some present with both. This variability in clinical presentation remains unexplained to this date.

Several non-surgical and surgical treatment options have been previously described. Mild symptoms or isolated hearing loss are preferably treated non-surgically. Physiotherapy and medication can improve mild symptoms. Surgery is reserved for serious complaints and appears to be most successful for vestibular symptoms and autophony. In addition to dehiscence, it may also be necessary to treat the provoking and concomitant pathologies. Surgical intervention can significantly improve the quality of life for these patients. Three surgical techniques can be used. The least invasive technique is transcanal round window reinforcement and tries to reduce the mobility of the round and oval windows. This technique has a good effect on vestibular complaints and autophony. The pseudo-conductive hearing loss is not affected. The major advantage of this technique is minimal invasiveness and the low risk of serious complications. Patients have a relatively high recurrence rate 1 year after the procedure [3].

A second technique-transmastoidal semicircular canal occlusion-involves final occlusion of the superior semicircular canal. This technique is sometimes used in treatment-resistant BPPV. Occlusion of the SCC prevents movement of the endolymph and ampulla of the SCCT and the function of the SCC is hereby lost. The loss of this function is mostly well tolerated. Loss of this function can be problematic in case of bilateral procedure, elderly patients and pre-existing loss of vestibular function. There is a risk of plugging the crus communis and losing both the superior and posterior semicircular canal, especially in large defects,. The major limitation is the restricted visibility of the dehiscence during the surgery. Also, anatomical variability occurs in patients with SCCD. Many of 
these patients have a lower position of the tegmen, leaving less room for dissection. The results are similar to those of the transcanal round window reinforcement [3] [11] [16] [17].

A third surgical option is a subtemporal craniotomy with an extradural approach to the eminentia arcuata and tegmen tympani. Once the dehiscence is visible, it is completely covered with different eligible materials, such as bone, fascia, cement, cartilage, implants. Some surgeons also plug the dehiscence. Pressure waves can no longer be exchanged between the inner ear and the intracranial cavity. The function of the SCC is hereby retained. The major advantages of this procedure are the high success rate on the vestibular complaints and autophony and the clear visibility on the defect, comparing to the other techniques [3] [16] [17]. This is also the only technique that can improve the reversible pseudo-conductive hearing loss, although there is no evidence for improvement [17]. This technique is obviously the most invasive technique. The main possible complication is insufficient coverage of dehiscence or secondary displacement, but early failure is rare. The risk of late failure is about $1 \%$. Another disadvantage is the presence of multiple small look-a-like defects in the counter potentially misleading the surgeon.

Several large studies demonstrate the high effectiveness of the plugging with/or resurfacing technique by using a subtemporal craniotomy as a treatment for debilitating complaints. Chung et al. [9] compared their own data with 7 other studies [10]-[17]. Complete recovery (100\% complaint-free) is achieved in $72 \%$ $100 \%$ of the patients, taking the variability between the studies into account [9]-[17]. Surgery is not advised in isolated hearing loss, as improvement of the hearing loss can't be guaranteed. In patients with solitary pressure-related symptoms, a tympanostomy may improve the complaints, although the patient's response can vary widely. Isolated tinnitus should not lead to surgical intervention, as the results on tinnitus are unpredictable. Still, there is a trend in which tinnitus would improve from 1 year postoperatively. Severe hearing loss is seen in $4 \%$ of patients postoperatively in any technique. In case of relapse, the complaints are often milder than before. Revision surgery can still improve the recurrence. Often recurrence is caused by retraction on the tissue plug, causing secondary motility. This is described as a "loose plug syndrome" and causes complaints according to the same principle as with primary dehiscence. Peripheral fascial palsy and vestibular complaints are mainly seen in the reinforcement and occlusion techniques. A study by Agrawal et al. showed vestibular hypofunction in $38 \%$ of patients after surgical resurfacing, with spontaneous improvement over 6 weeks (11\%) [10].

Gioacchini et al. [16] and Ziylan et al. [17] compared the different surgical techniques in their systematic reviews. No statistically significant difference could be found in the success rate and complications between the different possible techniques. However, there are limited publications and the surgical techniques used differ widely between individual surgeons. Their main conclusion was that surgical intervention greatly improves vestibular complaints and autophony in 
$95 \%$ and $90 \%$ of patients, respectively [16] [17].

Combinations of previous techniques use the advantages of both. Combining the transmastoid and subtemporal extradural approach, there is less brain retraction and better visibility of the defect. The main drawback is the compulsory mastoid oblitateration [3] [16] [17].

The variation in techniques and intraoperative variability between the different studies make it hard to compare the own data with literature, but our results seem to be similar to earlier reported studies. There has never a clear threshold described when to operate patient and when not to. All describe "severe complaints" as the reason for surgery, inducing a major potential difference in surgical threshold. More standardisation is necessary in future studies. Also, the result of the surgery depends particulary on the subjective improvement of the symptoms and the satisfaction of the patient.

Despite the small patient group, our strength lays in the reduced intraoperative variability by using the same team and the long term follow-up, suggesting a definitive result. We prefer the subtemporal extradural craniotomy, due to the high success rate, the direct view on the defect and the potential improvement of the reversible pseudo-conductive hearing loss [3] [16] [17].

The potential reversibilty of the pseudo-conductive hearing loss is noticed in all patients. Despite it is not guaranteed, it is only possible within the craniotomy technique. Recovery of the pseudo-conduction hearing loss was objectified in all 3 patients.

The definitive recovery of the conduction hearing loss and the absence of further symptoms surpass the invasive aspect of the procedure.

\section{Conclusion}

Surgical treatment should be considered in patients with severe, disabling DSSC symptoms. Different techniques can be considered depending on the type and severity of the complaints. The risks and invasiveness of each individual technique has to be taken into account. Surgical resurfacing of the DSSC via a subtemporal craniotomy has been confirmed as successful in multiple extensive studies. We were able to confirm the permanent result in all 3 patients. Our experience supports the vision of this safe and rewarding surgical technique as a treatment for debilitating DSSC. The definitive absence of symptoms and hearing recovery appear to outweigh the invasive aspect of the surgical technique.

\section{Conflicts of Interest}

The authors declare no conflicts of interest regarding the publication of this paper.

\section{References}

[1] Minor, L.B., Solomon, D., Zinreich, J.S. and Zee, D.S. (1998) Sound- and/or Pressure-Induced Vertigo Due to Bone Dehiscence of the Superior Semicircular Canal. Archives of Otorhinolaryngology-Head \& Neck Surgery, 124, 249-258. https://doi.org/10.1001/archotol.124.3.249 
[2] Carey, J.P., Minor, L.B. and Nager, G.T. (2000) Dehiscence or Thinning of Bone Overlying the Superior Semicircular Canal in a Temporal Bone Survey. Archives of Otorhinolaryngology-Head \& Neck Surgery, 126, 137-147. https://doi.org/10.1001/archotol.126.2.137

[3] Gianoli, G.J. and Soileau, J.S. (2017) Superior Semicircular Canal Dehiscence: Pathophysiology and Surgical Outcomes. Current Otorhinolaryngology Reports, 5, 153 159. https://doi.org/10.1007/s40136-017-0156-2

[4] Picavet, V., Govaere, F. and Forton, G. (2009) Superior Semicircular Canal Dehiscence: Prevalence in a Population with Clinical Suspected Otosclerosis-Type Hearing Loss. $B-E N T$, 5, 83-88.

[5] Minor, L.B. Carey, J.P., Cremer, P.D., Lustig, L.R., Streubel, S.O. and Ruckenstein, M.J. (2003) Dehiscence of Bone Overlying the Superior Canal as a Cause of Apparent Conductive Hearing Loss. Otology \& Neurotology, 24, 270-278. https://doi.org/10.1097/00129492-200303000-00023

[6] Neyt, P., Govaere, F. and Forton, G. (2011) Simultaneous True Stapes Fixation and Bilateral Bony Dehiscence between the Internal Carotid Artery and the Apex of the Cochlea: The Ultimate Pitfall. Otology \& Neurotology, 32, 909-913.

https://doi.org/10.1097/MAO.0b013e318225573f

[7] Mikulec, A., McKenna, M.J., Ramsey, M.J., Rosowski, J.J., Herrmann, B.S., Rauch, S.D., et al. (2004) Superior Semicircular Canal Dehiscence Presenting as Conductive Hearing Loss without Vertigo. Otology \& Neurotology, 25, 121-129. https://doi.org/10.1097/00129492-200403000-00007

[8] Tavassolie, T.S., Penninger, R.T., Zuñiga, M.G., Minor, L.B. and Carey, J.P. (2012) Multislice Computed Tomography in the Diagnosis of Superior Canal Dehiscence: How Much Error, and How to Minimize It? Otology \& Neurotology, 33, 215-222. https://doi.org/10.1097/MAO.0b013e318241c23b

[9] Chung, L.K., Ung, N., Spasic, M., Nagasawa, D.T., Pelargos, P.E., Thill, K., et al. (2016) Clinical Outcomes of Middle Fossa Craniotomy for Superior Semicircular Canal Dehiscence Repair. Journal of Neurosurgery, 125, 1187-1193. https://doi.org/10.3171/2015.8.JNS15391

[10] Agrawal, Y., Migliaccio, A.A., Minor, L.B. and Carey, J.P. (2009) Vestibular Hypofunction in the Initial Postoperative Period after Surgical Treatment of Superior Semicircular Canal Dehiscence. Otology \& Neurotology, 30, 502-506. https://doi.org/10.1097/MAO.0b013e3181a32d69

[11] Beyea, J.A., Agrawal, S.K. and Parnes, L.S. (2012) Transmastoid Semicircular canal Occlusion: A Safe and Highly Effective Treatment for Benign Paroxysmal Positional Vertigo and Superior Canal Dehiscence. The Laryngoscope, 122, 1862-1866. https://doi.org/10.1002/lary.23390

[12] Crane, B.T., Lin, F.R., Minor, L.B. and Carey, J.P. (2010) Improvement in Autophony Symptoms after Superior Canal Dehiscence Repair. Otology \& Neurotology, 31, 140-146. https://doi.org/10.1097/MAO.0b013e3181bc39ab

[13] Goddard, J.C. and Wilkinson, E.P. (2014) Outcomes Following Semicircular Canal Plugging. Otolaryngology_Head and Neck Surgery, 151, 478-483. https://doi.org/10.1177/0194599814538233

[14] Limb, C.J., Carey, J.P., Srireddy, S. and Minor, L.B. (2006) Auditory Function in Patients with Surgically Treated Superior Semicircular Canal Dehiscence. Otology \& Neurotology, 27, 969-980. https://doi.org/10.1097/01.mao.0000235376.70492.8e

[15] Niesten, M.E., McKenna, M.J., Grolman, W. and Lee, D.J. (2012) Clinical Factors Associated with Prolonged Recovery after Superior Canal Dehiscence Surgery. Otol- 
ogy \& Neurotology, 33, 824-831. https://doi.org/10.1097/MAO.0b013e3182544c9e

[16] Gioacchini, F.M., Alicandri-Ciufelli, M., Kaleci, S., Scarpa, A., Cassandro, E. and Re, M. (2016) Outcomes and Complications in Superior Semicircular Canal Dehiscence Surgery: A Systematic Review. The Laryngoscope, 126, 1218-1224. https://doi.org/10.1002/lary.25662

[17] Ziylan, F., Kinaci, A., Beynon, A.J. and Kunst, H.P. (2017) A Comparison of Surgical Treatments for Superior Semicircular Canal Dehiscence: A Systematic Review. Otology \& Neurotology, 38, 1-10. https://doi.org/10.1097/MAO.0000000000001277 